\title{
A case of possible familial Guillain-Barre syndrome
}

Familial Guillain-Barre syndrome (GBS) is rare. Occurrence in families and the association with HLA types have resulted in a genetic susceptibility being postulated $[1,2,3]$. In the absence of large scale epidemiological studies, isolated case reports of GBS within families support this hypothesis. We report a Sri Lankan father and daughter, who shared HLA types DR12 and DQ 6, 7 and developed GBS eleven months apart.

A 7-year old girl developed sudden onset lower limb weakness progressing to involve upper limbs. Within three days, she was unable to walk even with support. There was no ataxia, bladder or bowel involvement. Ten days prior to this illness she had bloody diarrhoea and stool culture grew Campylobacter jejuni. On examination she was conscious and oriented. Cranial nerves were normal. There was generalised hypotonia, lower limbs were arreflexic with grade 2 muscle power; upper limbs were hyporeflexic with grade 4 power. There was no sensory or autonomic involvement. On day 12 of the illness cerebrospinal fluid analysis was normal. Nerve conduction was compatible with acute motor axonal neuropathy (AMAN) type GBS. HLA typing found DR 12, 15, 51 and 52 and DQ 6, 7. Over the next six months she made a complete recovery.

Eleven months previously, her father, aged 51 years, had developed back pain, numbness and weakness in lower limbs, progressing rapidly to loss of ambulation.
Medical records showed that muscle power was grade 0 in lower limbs and grade 3 in upper limbs. Unilateral lower motor neuron type 7th nerve palsy was noted. Nerve conduction studies showed an acute inflammatory demyelinating polyneuropathy (AIDP) and cerebrospinal fluid showed a cytoprotein dissociation. HLA typing showed DR 12, 13, 52 and DQ 6 and 7. He received intravenous immunoglobulins and recovered completely by the time his daughter fell ill. There was no family history to support hereditary neuropathy with liability to pressure palsy.

Most reported cases of familial GBS are among Caucasians. The only previous case report from Asia we found was of two siblings of Japanese parentage who developed GBS within a few days suggesting an infective rather than a familial aetiology [4]. The largest reported survey for a familial incidence in Netherlands described twelve affected families. Common HLA types have been studied without a definitive association being established. We found HLA types DR12 and DQ 6, 7 to be identical in this father and daughter pair.

\section{References}

1. Geleijns K, Brouwer BA, Jacobs BC, HouwingDuistermaat JJ, van Duijn CM, van Doorn PA. The occurrence of Guillain-Barre syndrome within families. Neurology 2004; 63: 1747-50. 
2. Wilmshurst JM, Pohl KR, Vaughan RW, et al. Familial Guillain-Barre syndrome. European Journal of Neurology 1999; 6: 499-503.

3. Gorodesky C, Varela B, Castro-Escobar LE, Chaves-Negrete A, Escobar-Gutierrez, Martinez-Mata J. HLA antigens in
Mexican patients with Guillaine-Barre syndrome. Journal of Immunology 1983; 4: 1-7.

4. Yuki NY, Tsujino F. Familial Guillain-Barre syndrome subsequent to Campylobacter jejuni enteritis. Journal of Paediatrics 1995; 126: 162.

\section{P Senanayake ${ }^{1}$, J Wanigasinghe ${ }^{2}$, N Gamaethige ${ }^{3}$, P Dissanayake $^{4}$}

${ }^{1}$ Department of Paediatrics, Faculty of Medicine, University of Colombo, ${ }^{2}$ Neurology Unit and ${ }^{3}$ Paediatrics Unit, Lady Ridgeway Hospital, Colombo, and ${ }^{4}$ Medical Unit, National Hospital of Sri Lanka.

Correspondence: MPS, e-mail <manouri.senanayake@gmail.com>. Competing interests: none declared. 